\title{
Molecular simulations of the adsorption of cycloalkanes in MFI-type silica
}

\author{
Merijn Schenk, ${ }^{a} a$ Berend Smit ${ }^{b c}$ Theo L. M. Maesen ${ }^{d}$ and Thijs J. H. Vlugt ${ }^{e}$ \\ ${ }^{a}$ Laboratory of Physical Chemistry, Swiss Federal Institute of Technology, ETH-Hönggerberg, \\ 8093 Zürich, Switzerland.E-mail:merijn@igc.phys.chem.ethz.ch;Tel: +41-1-6334239 \\ ${ }^{\boldsymbol{b}}$ Van't Hoff Institute for Molecular Sciences, University of Amsterdam, Nieuwe Achtergracht \\ 166, $1018 \mathrm{WV}$ Amsterdam, The Netherlands \\ ${ }^{c}$ CECAM (Centre Européen de Calcul Atomique et Moléculaire), Ecole Normale Supérieure, \\ 46 Allée d'Italie, 69364 Lyon Cedex 7, France \\ ${ }^{d}$ ChevronTexaco, Energy Technology Company, Chevron Way 100, Richmond, California, \\ 94802-0627, USA \\ ${ }^{\boldsymbol{e}}$ Condensed Matter and Interfaces, Debye Institute, Utrecht University, P.O. Box 80000, \\ 3508 TA Utrecht, The Netherlands
}

Received 18th March 2005, Accepted 19th May 2005

First published as an Advance Article on the web 1st June 2005

\begin{abstract}
A new force field for the simulation of the adsorption of cycloalkanes in nanoporous silica affords a significant improvement over any previously employed force field. The simulated isotherms reproduce the most salient features in the experimental isotherms extremely well. The study of cyclo-pentane, -hexane, and -heptane adsorption in MFI-type silica indicates an inflection for cyclopentane but not for cyclohexane at intermediate pressure. If corroborated by experiments, such an inflection point would afford an excellent calibration point for further force field developments. At low pressures, mixture isotherms of cyclohexane and $n$-hexane show a temperature dependence on the selectivity in accordance with recent results by J. P. Fox and S. P. Bates, J. Phys. Chem., 2004, 108, 17136. This dependence is caused by a difference in temperature dependence of the Henry coefficient for both molecules. At high pressures entropy effects due to packing always favor the sorption of $n$-hexane. Furthermore, the influence of the flexibility of the zeolite framework on the adsorption of these rather bulky molecules is investigated. It is found that this influence of the flexibility on the adsorption of cyclohexane is as small as with $n$-alkanes.
\end{abstract}

\section{Introduction}

Zeolites are microporous crystalline materials, built up from $\mathrm{TO}_{4}$ tetrahedral units, where the central $\mathrm{T}$ atom is usually silicon or aluminium. The units are linked through the oxygen atoms, creating three-dimensional networks which, depending on the type of zeolite structure, define cylindrical or cage-like pores. These pores may be linked in a one-, two-, or threedimensional way, and are often big enough to allow small molecules, like alkanes or water, to enter. Because of their special structure and properties, there are several applications of zeolites in the petrochemical industries, such as (selective) adsorption, and catalysis.

Knowledge of the adsorption behavior of hydrocarbons in the pores of zeolites is of considerable importance for our understanding of the performance of zeolites in these applications. $^{1-4}$ This adsorption behavior is usually quantified by means of the adsorption isotherm, which is the quantity of hydrocarbons adsorbed at a given temperature and pressure. Experimentally, the determination of adsorption isotherms can be quite time consuming, because of the slow diffusion of hydrocarbons in the pores of a zeolite. ${ }^{5}$ When mixtures of hydrocarbons are considered, experiments become increasingly complicated. Measurements at the conditions of interest often require a complicated experimental setup (due to high temperature and pressure), and often unwanted side-effects like chemical reactions intrude. Therefore, computer simulations can provide a valuable alternative to obtain adsorption isotherms.
In the past few years, the adsorption and diffusion of hydrocarbons in zeolites have been studied extensively using computer simulations. ${ }^{6-23}$ In many of these studies, only linear, branched and aromatic hydrocarbons were considered. Only four studies, two by Snurr et al. ${ }^{24,25}$ and two by Fox et al., ${ }^{26,27}$ include cycloalkanes (naphthenes).

Despite a major interest in the adsorption of naphthenes in the petrochemical and refinery industries, there is -as yet- no major simulation effort. Most industrial feeds usually contain predominantly naphthenes and aromatics. An understanding at a molecular level of the adsorption and conversion of naphthenes (and aromatics) similar to that obtained for paraffins would significantly enhance the understanding of many refinery processes. Naturally, naphthenes fit more tightly inside zeolite topologies that can readily accommodate paraffins. This can result in slow diffusion, which makes experiments difficult. From a theoretical point of view, tight fitting molecules are interesting because they allow the study of the influence of zeolite framework flexibility on the adsorption isotherms. Previous studies found ${ }^{29}$ that this influence is rather small for mono-branched alkanes in MFI-type silica. Furthermore, the size of the molecules makes the sampling of the phase space in the simulations difficult. Therefore it is important to investigate efficient sampling schemes, which may additionally be applied to other bulky molecules, like aromatics.

A prerequisite for obtaining meaningful results from molecular simulations is an adequate description of the interactions. An additional motivation to conduct the present study is therefore to derive a set of interaction parameters to model 


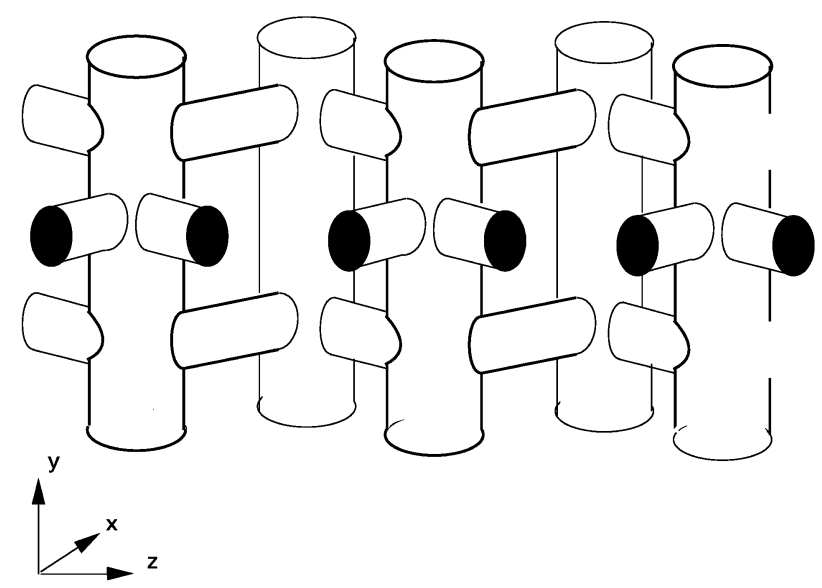

Fig. 1 Schematic representation of MFI-type silica. Straight channels ( $y$ direction) and zigzag channels (in the $x-z$ plane) cross each other at the intersections.

the adsorption of cycloalkanes in nanoporous silica and alumophosphates. We opted to start with MFI-type silica because it has been the subject of many experimental and computational studies, and because it was shown previously ${ }^{30,39}$ that potential parameters derived for MFI can be readily used to simulate adsorption in other silica and alumophosphate topologies. The MFI topology has two channel types, the zigzag and the straight channels that cross at the intersection; see Fig. 1.

This paper is organized as follows. In the next section, we describe the interaction potentials used in the simulations, and in Section 3 we give an overview of our simulation technique. In Section 4 we first assess the accuracy of existing sets of zeolite-alkane interaction parameters and subsequently develop a new, more accurate set of parameters. We then continue to report adsorption isotherms, Henry coefficients and heats of adsorption calculated in both rigid and flexible zeolite frameworks using the new parameters. Furthermore, we report adsorption isotherms of an equimolar mixture of linear and cyclic $\mathrm{C}_{6}$. We summarize our findings in Section 5 .

\section{Model}

The model to describe the cycloalkane molecules is largely taken from the work of Neubauer et al. ${ }^{31}$ because this model can satisfactorily reproduce vapor-liquid equilibria. Since we made some minor changes to the model (modifying the functional form of the stretching-, bending- and torsion-potentials), it is described below.

The Neubauer model describes the cycloalkanes using a united atom representation, i.e. it models $\mathrm{CH}_{2}$ groups as single interaction centers. For the molecules considered in this study, there are only intermolecular interactions between these pseudoatoms. A Lennard-Jones potential describes these interactions as follows

$$
u^{\mathrm{LJ}}\left(r_{i j}\right)=4 \varepsilon\left[\left(\frac{\sigma}{r_{i j}}\right)^{12}-\left(\frac{\sigma}{r_{i j}}\right)^{6}\right]
$$

where $r_{i j}$ is the distance between atoms $i$ and $j$. For cyclohexane and cycloheptane, the size parameter $\sigma_{\mathrm{CH}_{2}}=3.93 \AA$ and the energy parameter $\varepsilon_{\mathrm{CH}_{2}} / k_{\mathrm{B}}=50.37 \mathrm{~K}$, while for cyclopentane $\sigma_{\mathrm{CH}_{2}}=3.85 \AA$ and $\varepsilon_{\mathrm{CH}_{2}} / k_{\mathrm{B}}=50.5 \mathrm{~K}$. The Lennard-Jones potential is truncated at $13.8 \AA$, and the usual tail corrections are applied. ${ }^{32}$ Bond-stretching is modeled by a harmonic potential

$$
u^{\text {stretch }}\left(r_{i j}\right)=\frac{1}{2} k_{s}\left(r_{i j}-r_{0}\right)^{2}
$$

with an equilibrium bond-length $r_{0}$ of $1.532 \AA$ and a force constant $k_{s} / k_{\mathrm{B}}$ of $96500 \mathrm{~K}^{-2}$. Bond-bending is modeled by a harmonic cosine potential

$$
u^{\text {bend }}(\theta)=\frac{1}{2} k_{\theta}\left[\cos (\theta)-\cos \left(\theta_{\mathrm{eq}}\right)\right]^{2}
$$

where the equilibrium angle $\theta_{\mathrm{eq}}=111.8^{\circ}$ and the force constant $k_{\theta} / k_{\mathrm{B}}=85000 \mathrm{~K} \mathrm{rad}^{-2}$. The torsional angles are controlled by

$$
u^{\text {tors }}(\phi)=\sum_{i=0}^{i=5} C_{i} \cos ^{i}(\phi)
$$

where $C_{1} / k_{\mathrm{B}}=1204.654 \mathrm{~K}, C_{2} / k_{\mathrm{B}}=1947.740 \mathrm{~K}, C_{3} / k_{\mathrm{B}}=$ $-357.845 \mathrm{~K}, C_{4} / k_{\mathrm{B}}=1944.666 \mathrm{~K}, C_{5} / k_{\mathrm{B}}=715.690 \mathrm{~K}$, and $C_{6} / k_{\mathrm{B}}=-1565.572 \mathrm{~K}$.

In this study we consider both rigid and flexible zeolite frameworks. In both cases we assume that the interactions between the alkanes and the zeolite are governed by the dispersive interactions with the oxygen atoms. These interactions are described by a Lennard-Jones potential, for which the parameters are fitted in the Results and discussion section. The Lennard-Jones potential is truncated at $13.8 \AA$, and the usual tail corrections are applied. ${ }^{32}$

The model which describes the movement of the zeolite atoms in the flexible framework is a modified version of the model by Demontis and co-workers. ${ }^{28}$ In this model, which is conceptually the most simple model available, harmonic potentials are used between $\mathrm{Si}-\mathrm{O}$ and $\mathrm{O}-(\mathrm{Si})-\mathrm{O}$ bonds,

$$
\begin{aligned}
& V_{\mathrm{Si}-\mathrm{O}}(r)=k_{\mathrm{Si}-\mathrm{O}} \times\left(r-r_{0}, \mathrm{Si-O}\right)^{2} \\
& V_{\mathrm{O}-\mathrm{O}}(r)=k_{\mathrm{O}-\mathrm{O}} \times\left(r-r_{0}, \mathrm{O}-\mathrm{O}\right)^{2}
\end{aligned}
$$

in which $r_{0, \mathrm{Si}-\mathrm{O}}$ and $r_{0, \mathrm{O}-\mathrm{O}}$ are the equilibrium bond lengths, taken directly from the crystal structure, and $k_{\mathrm{Si}-\mathrm{O}}$ and $k_{\mathrm{O}-\mathrm{O}}$ the spring constants. Only nearest neighbors are considered, and there are no direct $\mathrm{Si}-\mathrm{Si}$ interactions. The flexibility of the zeolite framework can be controlled by varying the $k$ 's, where the crystal structure is reproduced exactly when $T \rightarrow 0$ or $k \rightarrow \infty$. To reduce the number of parameters, we have chosen $k=k_{\mathrm{O}-\mathrm{O}}=k_{\mathrm{Si}-\mathrm{O}} / 5$ (which is approximately the case for the original Demontis model). Details of this modified model can be found in ref. 29.

\section{Simulation technique}

All simulations are performed using the Monte Carlo simulation technique. The Henry coefficients (the slope of the adsorption isotherm at very low loading) and the heat of adsorption are calculated in the NVT ensemble, using a single molecule. The adsorption isotherms are calculated in the grand-canonical $(\mu \mathrm{VT})$ ensemble. In the figures, wherever the simulated adsorption isotherms are compared to experimental isotherms, we have used the Redlich-Kwong equation of state to convert the applied chemical potential $\mu$ to pressures and stopped the axis labeling above the saturation vapor pressure. In all other cases, the applied chemical potential is given as fugacity.

The molecules are inserted from a reservoir containing an ideal gas of cyclic molecules, in a similar way as other authors. ${ }^{33-35}$ Before each trial insertion the conformation of a molecule is changed through a small Monte Carlo run, long enough to ensure independent configurations with the correct internal distribution. The trial moves in this run consist of atom translations and crankshaft moves. A crankshaft move rotates an atom around an axis running through its neighboring atoms. This move is especially efficient in changing the torsion angles. This small Monte Carlo run ensures a correct distribution of cyclohexane conformations (we do not fix the trial molecules in either boat or chair form). After the conformation change, the molecule is given a random orientation and is inserted into the zeolite. Since the probability of inserting a complete cycloalkane molecule in one move is very low, we use the multiple first bead scheme. ${ }^{36}$ In this scheme, many 
(in our case 15) possible positions for one of the atoms of the molecule are created. One of them is selected with a probability according to its Boltzmann weight and subsequently the rest of the trial molecules are inserted relative to the position of this first atom. This scheme greatly improves the probability of inserting a molecule, because it ensures that relatively good starting positions for the trial molecules are found.

Furthermore, trial moves are performed to translate or rotate previously inserted molecules. The total number of trial moves is of the order of $10^{6}$ for equilibration and another $10^{6}$ for sampling. In the simulations using a flexible zeolite, additional trial moves are performed to displace the zeolite atoms. In this case, the total number of trial moves for both equilibration and sampling is of the order of $10^{9}$. For more details, we would like to refer the reader to refs. 12 and 39.

\section{Results and discussion}

To verify whether the interactions between the $\mathrm{CH}_{2}$ groups of cyclohexane and the zeolite framework can be modeled using the parameters for the $\mathrm{CH}_{2}$ groups of linear alkanes, we calculated an adsorption isotherm using the parameters from Vlugt et al. ${ }^{12}$ (see Table 1). As is shown in Fig. 2, these parameters do not reproduce the experimental isotherm of Cavalcante et al. $^{41}$

As the Neubauer ${ }^{31}$ gas-phase parameters for the cyclohexane $\mathrm{CH}_{2}$ groups differ from the gas-phase parameters for the $\mathrm{CH}_{2}$ groups of linear alkanes from the widely used TraPPE ${ }^{40}$ alkane forcefield, we scaled the Vlugt et al. parameters for the interaction with the zeolite accordingly (first only $\varepsilon$, after that both $\sigma$ and $\varepsilon$; see Table 1). The calculated adsorption isotherms using these scaled parameters is also shown in Fig. 2. As can be seen, although better, these also largely deviate from the experimental isotherm.

Two previous studies reported the successful application of a set of parameters, one by Snurr et al. ${ }^{24}$ (parameters by June et $a l^{6}{ }^{6}$ ) and one by Fox et al. ${ }^{26}$ As both sets differ significantly (see Table 1), we used both to calculate an adsorption isotherm of cyclohexane in MFI-type silica at two temperatures and compared them to experiments of Song et al. ${ }^{42}$ and Cavalcante et al. ${ }^{41}$ The results are listed in Figs. 3 and 4 and Table 2. Although applied successfully in their own study, the Fox parameters do not seem to give accurate results in our simulations. On closer inspection, the parameters of Fox et al. show the smallest size parameter $\sigma$. A size parameter that is too small can be compensated through a larger value of $\varepsilon$ (see Fig. 5). However, the value of $\varepsilon$ in the Fox parameter set is also very low compared to the other parameter sets. The parameters of June et al. give a considerably better agreement with experiment. It is important to note that we obtain the same results as reported by Snurr et al. using their parameters.

The models listed earlier are not accurate enough to capture all details of the adsorption behavior of cycloalkanes, so that new parameters have to be fitted. It is important to note at this point that both the parameter set of Snurr et al. and of Fox

Table 1 Lennard-Jones parameters describing the interactions between the $\mathrm{CH}_{2}$ groups of the cycloalkanes and the zeolite oxygen atoms. The parameters for the $n$-alkane $\mathrm{CH}_{2}$ groups were taken from Vlugt et al. ${ }^{12}$

\begin{tabular}{lll}
\hline & $\varepsilon k_{\mathrm{B}}^{-1} / \mathrm{K}$ & $\sigma / \AA$ \\
\hline$n$-alkane ${ }^{12}$ & 58.0 & 3.60 \\
Scaled eps & 63.5 & 3.60 \\
Scaled eps + sig & 63.5 & 3.51 \\
Snurr et al. $(\mathrm{June} \text { et } \text { al. })^{24}$ & 83.8 & 3.364 \\
Fox et al. $^{26}$ & 66.0 & 3.30 \\
This work & 69.0 & 3.55 \\
\hline
\end{tabular}

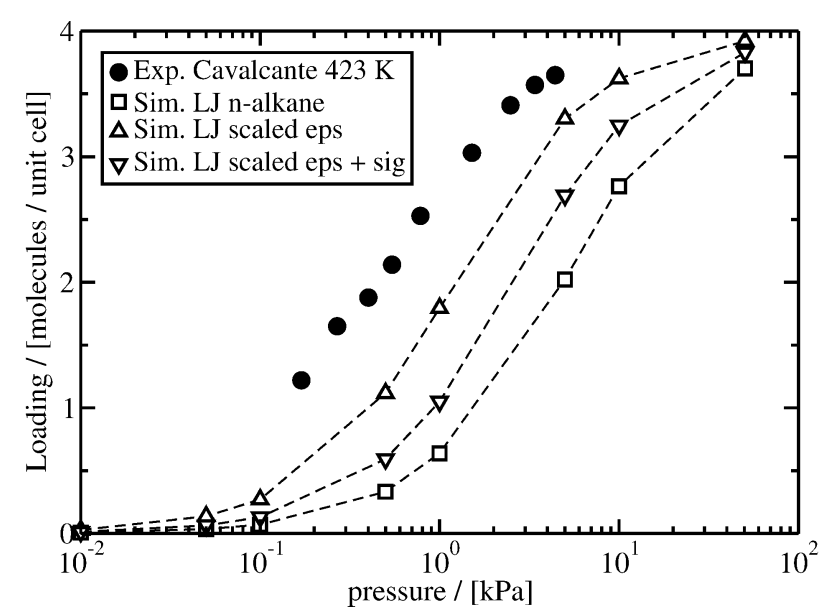

Fig. 2 Comparison of simulated adsorption isotherms of cyclohexane in MFI-type silica to experimental data of Cavalcante et al. at $T=423$ $\mathrm{K} .{ }^{41}$ The simulations were performed using the Lennard-Jones parameters for the $\mathrm{CH}_{2}$ group of $n$-alkanes by Vlugt et al. ${ }^{12}$ and scaled parameters respectively (see Table 1). The lines serve as a guide to the eye.

et al. predict an inflection in the isotherm at 4 molecules per unit cell. We will discuss this observation later on in this paper.

The aim is to obtain a set of Lennard-Jones parameters which enable an accurate calculation of adsorption isotherms over a wide range of temperatures and pressures. As was shown in ref. 43, a good starting point is to find a set of Lennard-Jones parameters that faithfully predict the adsorption properties at low loading, i.e. both the Henry coefficient and the heat of adsorption should be reproduced. To find these parameters, we performed a series of simulations where for a given size parameter $\sigma$ we changed the energy parameter $\varepsilon$ until the experimental values were recovered. Both the Henry coefficient and the heat of adsorption, fitted to experimental data from Cavalcante et al., are given in Fig. 5. Where the lines cross, both the Henry coefficient and the heat of adsorption are correctly reproduced by the same, unique, set of parameters. The optimum values for the $\mathrm{CH}_{2}^{\text {cyclo }}-\mathrm{O}^{\text {zeo }}$ interaction parameters are $\sigma=3.55 \AA$ and $\varepsilon / k_{\mathrm{B}}=70.0 \mathrm{~K}$. As it turns out, better overlap with the isotherms is achieved with a slightly lower $\varepsilon / k_{\mathrm{B}}$ of $69.0 \mathrm{~K}$. This is probably because the reported Henry coefficients and heats of adsorption by Cavalcante et al. are derived from fits to their measured isotherms. Therefore, small

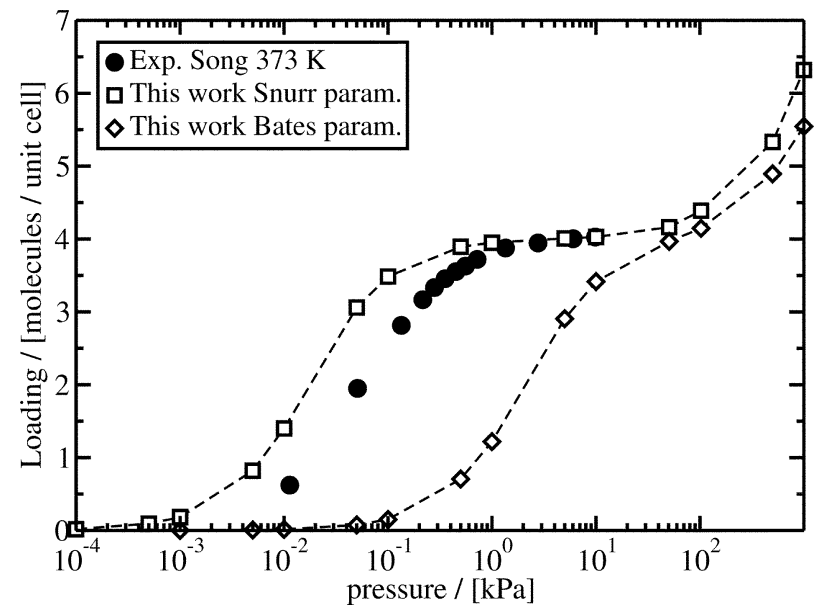

Fig. 3 Comparison of simulated adsorption isotherms of cyclohexane in MFI-type silica to experimental data of Song et al. at $T=373 \mathrm{~K}^{42}$ The simulations were performed using the Lennard-Jones parameters as used by Snurr et al. (June parameter set) ${ }^{24}$ and Fox et al. ${ }^{26}$ (see Table 1). The lines serve as a guide to the eye. 


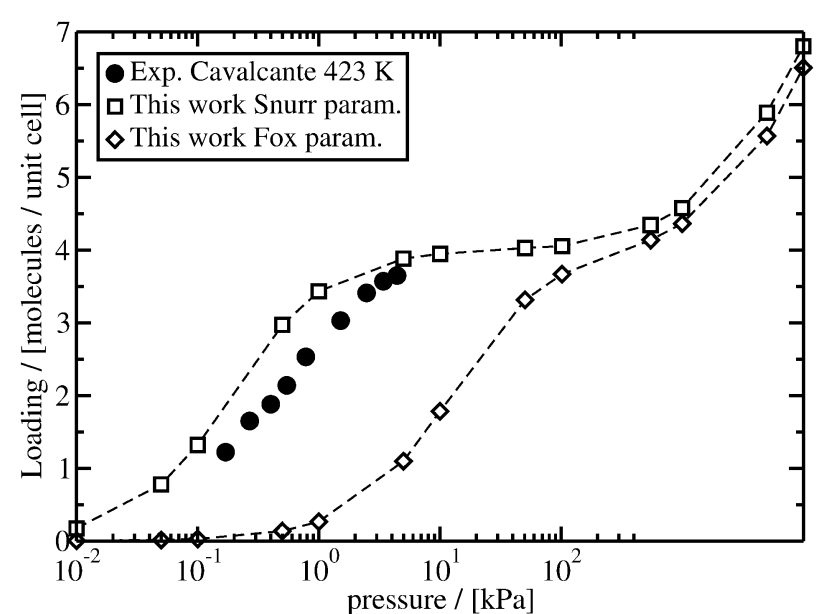

Fig. 4 Comparison of simulated adsorption isotherms of cyclohexane in MFI-type silica to experimental data of Cavalcante et al. at $T=423$ $\mathrm{K} .{ }^{41}$ The simulations were performed using the Lennard-Jones parameters as used by Snurr et al. (June parameter set) ${ }^{24}$ and Fox et al. ${ }^{26}$ (see Table 1). The lines serve as a guide to the eye.

errors in these fits propagate into the Lennard-Jones parameters.

To understand the effect of this small alteration of $\varepsilon$, we recalculated the Henry coefficient and the heat of adsorption using $\sigma=3.55 \AA$ and $\varepsilon / k_{\mathrm{B}}=69.0 \mathrm{~K}$. The results, given in Table 2 , show that these new parameters still give a good agreement.

Using these new parameters we calculated the adsorption isotherm of cyclohexane in MFI-type silica for 6 different temperatures, and compared them to experimental data from Cavalcante et al. and Song et al. Figs. 6 and 7 show that the simulations are in excellent agreement with the experimental results, except for the lowest temperature in both sets. We attribute both deviations to experimental difficulties, because of the following. In both cases (Song at $T=323 \mathrm{~K}$ and Cavalcante at $T=393 \mathrm{~K}$ ) the maximum loading is higher than 4 molecules per unit cell. This is in itself not unusual, but in both cases the loading overshoots the 4 molecules per unit cell without an inflection in the isotherm. Many studies, both experimental and computational, have shown that the isotherms of bulky molecules, like isobutane, ${ }^{11,12,37,38}$ in MFItype silica show an inflection as the molecules need an extra "push" to adsorb in the narrow channels once the 4 intersections per unit cell are occupied. The overshoot can also not be temperature related, since the highest temperature of Song et al. is still lower than the lowest temperature of Cavalcante et al., the former being correctly reproduced.

To verify whether the adsorption isotherm of cyclohexane in MFI-type silica shows an inflection, as was predicted by the other parameter sets, we performed a simulation at $T=300 \mathrm{~K}$ over a wide range of pressures. As can be seen in Fig. 8 this is not the case up to $10^{5} \mathrm{kPa}$ at $T=300 \mathrm{~K}$.

In addition, we simulated the adsorption isotherms of cyclopentane and cycloheptane in MFI-type silica using the new parameters derived for cyclohexane. For both molecules

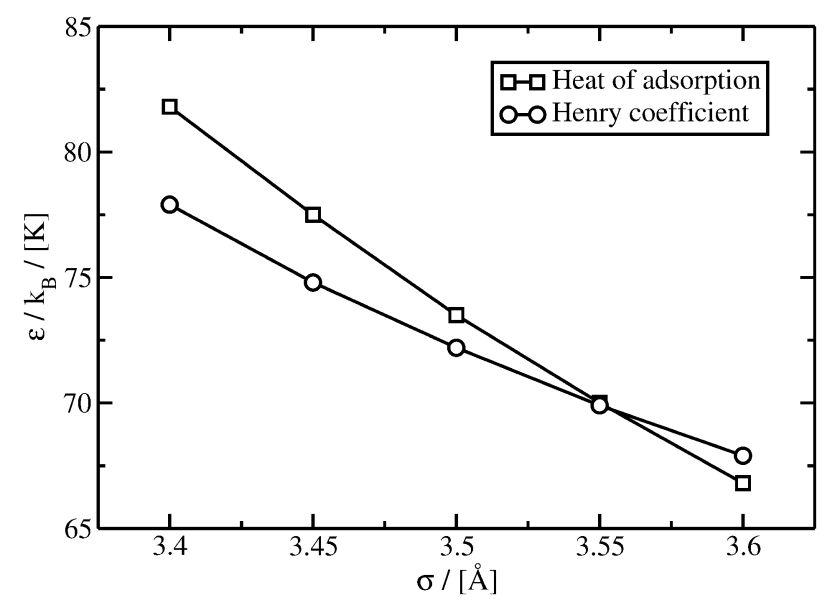

Fig. 5 The Lennard-Jones parameters for which the heat of adsorption and Henry coefficient of cyclohexane in MFI-type silica agree with the experimental results of Cavalcante et al. at $T=423 \mathrm{~K} .{ }^{41}$ The lines cross at the unique set which reproduces both the heat of adsorption and the Henry coefficient.

there are, to the best of our knowledge, no experimental data available. The results are shown in Figs. 9 and 10 respectively. As can be expected on the basis of their molecular size, the isotherms of cyclopentane show an inflection at 4 molecules per unit cell, while the isotherms for the larger cycloheptane do not.

Recently, it was shown that interaction parameters can be fitted to a high degree of accuracy using inflection points in isotherms. ${ }^{44}$ As the simulations with a fixed framework as well as the available experimental results do not show inflection behavior, the interaction parameters can not be further finetuned using this technique. Naturally, also experimental results for cyclopentane would be valuable for validating the newly derived parameters.

For many practical applications, it is of interest to know the adsorption behavior of mixtures of hydrocarbons. As a first exploration into this area involving cyclohexane molecules, we therefore simulated the adsorption of an equimolar mixture of cyclohexane and $n$-hexane at two different temperatures. The results are given in Figs. 11 and 12.

The sorption behavior of mixtures is determined by the interplay between the number and strength of the sorption sites of each molecule, and packing effects within the channels. ${ }^{4,10,12,45}$ From the simulated isotherms at $300 \mathrm{~K}$ up to high pressure (Fig. 8), it was clear that cyclohexane can only adsorb at the intersections. This is in contrast to $n$-hexane, which can adsorb throughout the entire pore system. ${ }^{7,12}$ The results for the mixture show that at $300 \mathrm{~K}, n$-hexane is preferentially adsorbed at all pressures, while only a small portion of cyclohexane is adsorbed at lower pressures. At higher pressures $n$-hexane completely expels cyclohexane, because of configurational entropy effects; $n$-hexane molecules pack more easily inside the MFI-type framework, because they can be located anywhere, whereas cyclohexane is restricted to the

Table 2 Comparison between the experimental Henry coefficients and heats of adsorption of Cavalcante et $_{\text {al }}{ }^{41}$ at $T=423 \mathrm{~K}$ and $473 \mathrm{~K}$ and those calculated by us using the Lennard-Jones parameters of Snurr et al. ${ }^{24}$ (parameters by June et al. ${ }^{6}$ ), Fox et al., ${ }^{26}$ and this work

\begin{tabular}{|c|c|c|c|c|}
\hline \multirow[b]{2}{*}{ LJ set } & \multicolumn{2}{|l|}{$T=423 \mathrm{~K}$} & \multicolumn{2}{|l|}{$T=473 \mathrm{~K}$} \\
\hline & $q_{\text {ads }} / \mathrm{kJ} \mathrm{mol}^{-1}$ & $K_{\mathrm{h}} / \mathrm{mmol} \mathrm{g}^{-1} \mathrm{~Pa}^{-1}$ & $q_{\text {ads }} / \mathrm{kJ} \mathrm{mol}^{-1}$ & $K_{\mathrm{h}} / \mathrm{mmol} \mathrm{g}^{-1} \mathrm{~Pa}^{-1}$ \\
\hline Snurr et al. (June et al. $)^{24}$ & -62.0 & $3.09 \times 10^{-3}$ & -62.1 & $4.77 \times 10^{-4}$ \\
\hline Fox et $a .^{26}$ & -45.3 & $4.80 \times 10^{-5}$ & -45.4 & $1.22 \times 10^{-5}$ \\
\hline This work & -62.1 & $1.23 \times 10^{-3}$ & -61.9 & $1.90 \times 10^{-4}$ \\
\hline Exp. Cavalcante et al. ${ }^{41}$ & -63.2 & $1.52 \times 10^{-3}$ & -63.2 & $1.78 \times 10^{-4}$ \\
\hline
\end{tabular}




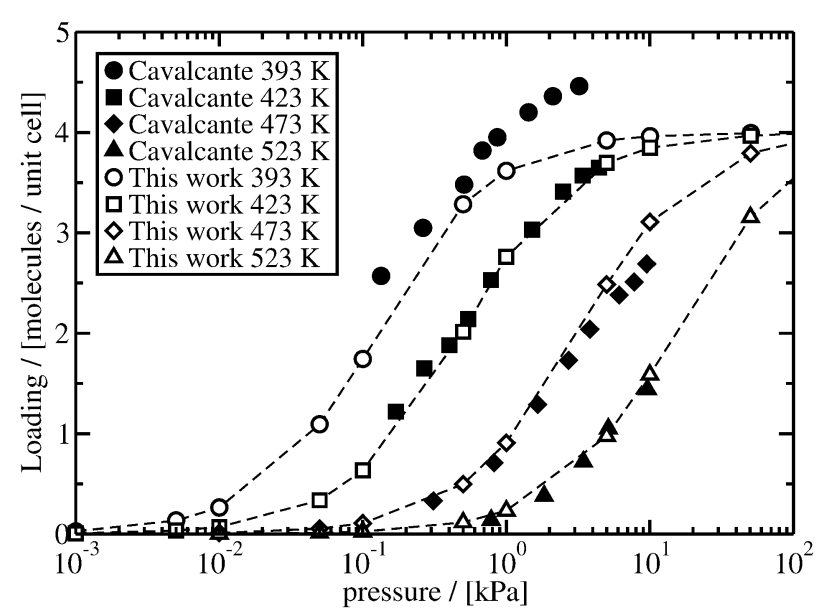

Fig. 6 Comparison of simulated adsorption isotherms of cyclohexane in MFI-type silica to experimental data of Cavalcante et al. ${ }^{41}$ In these simulations the newly fitted parameters are used (Table 1). The lines serve as a guide to the eye.

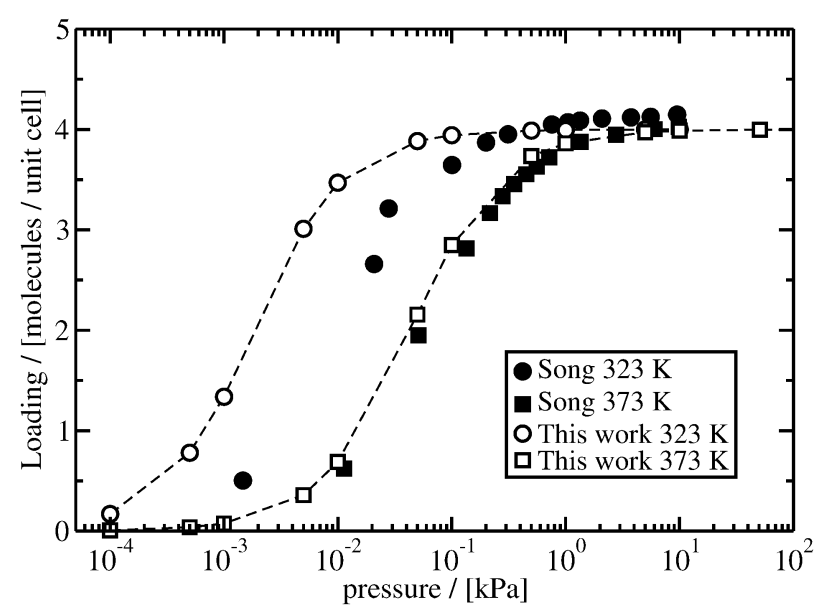

Fig. 7 Comparison of simulated adsorption isotherms of cyclohexane in MFI-type silica to experimental data of Song et $a l^{42}$ In these simulations the newly fitted parameters are used (Table 1). The lines serve as a guide to the eye.

intersections. Only at $600 \mathrm{~K}$ and low pressure do we observe a selectivity for cyclohexane. This change in selectivity with temperature at low pressure was also observed recently by Fox et al., ${ }^{27}$ and reflects a change in the Henry coefficient with temperature (see Fig. 13).

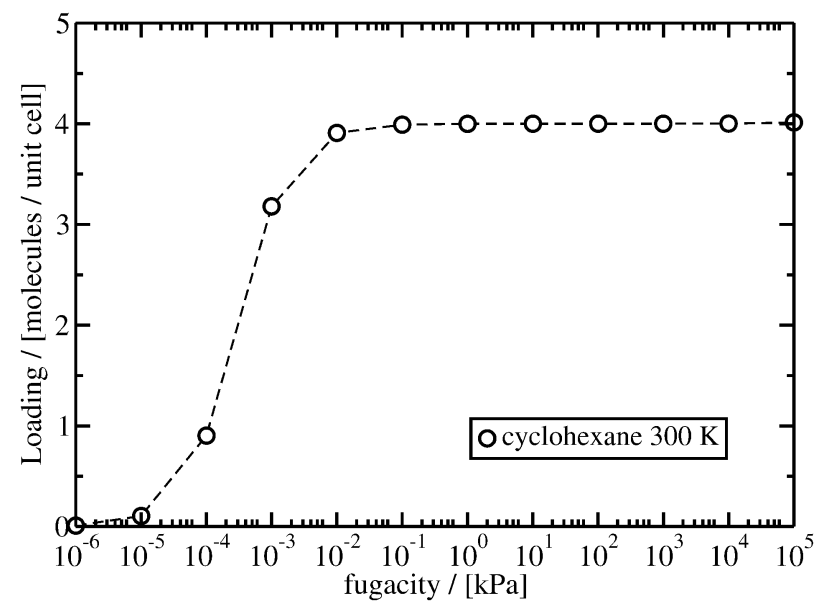

Fig. 8 Simulated adsorption isotherm of cyclohexane in MFI-type silica at $T=300 \mathrm{~K}$, using the newly fitted parameters (Table 1). No inflection is observed, even at extremely high pressures. The lines serve as a guide to the eye.

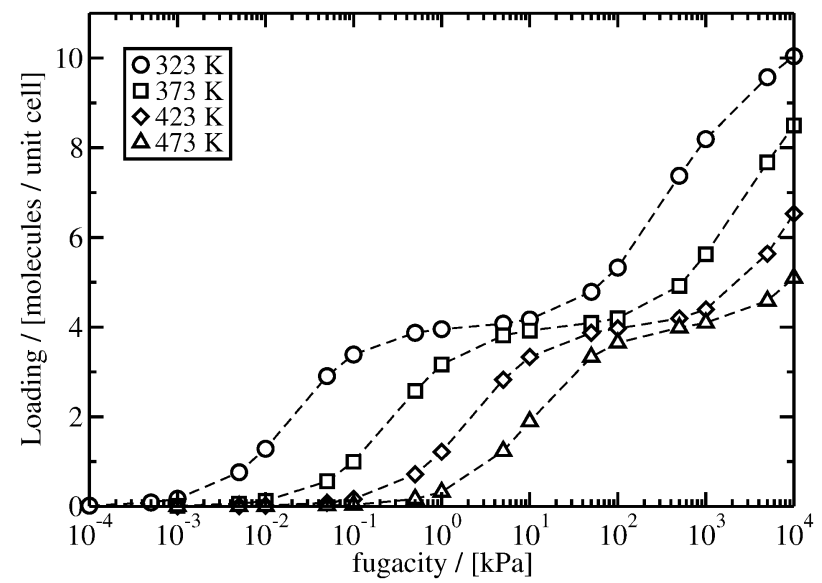

Fig. 9 Simulated adsorption isotherms of cyclopentane in MFI-type silica, using the newly fitted parameters (Table 1). The lines serve as a guide to the eye.

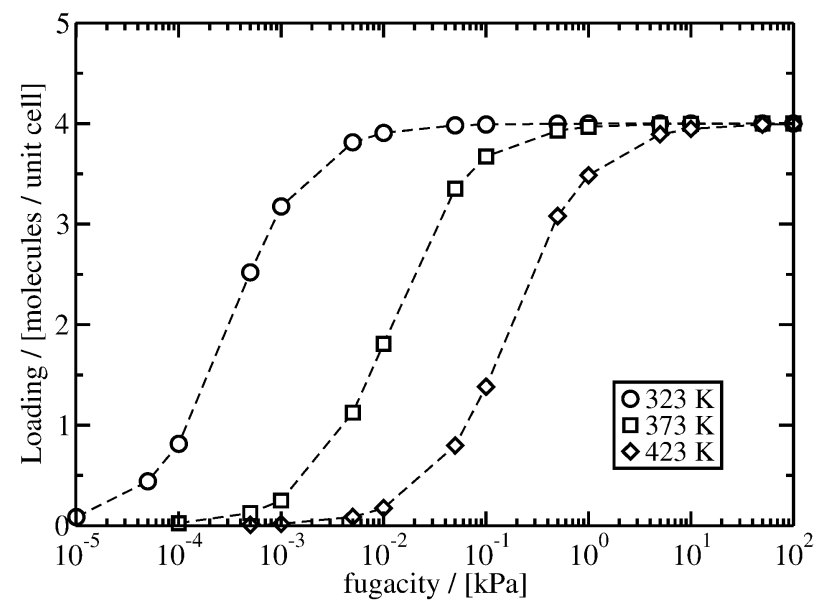

Fig. 10 Simulated adsorption isotherms of cycloheptane in MFI-type silica, using the newly fitted parameters (Table 1). The lines serve as a guide to the eye.

Since the Henry coefficient is closely related to the free energy of adsorption, we can analyze the energetic and entropic contributions to the difference in temperature dependence of the Henry coefficient. In Fig. 14 the difference in free energy of adsorption $(\Delta \Delta G)$ between cyclohexane and $n$-hexane, as well as the difference in adsorption enthalpy $(\Delta \Delta H)$ and adsorption entropy $(\Delta \Delta S)$ is given. To ease viewing, the individual graphs

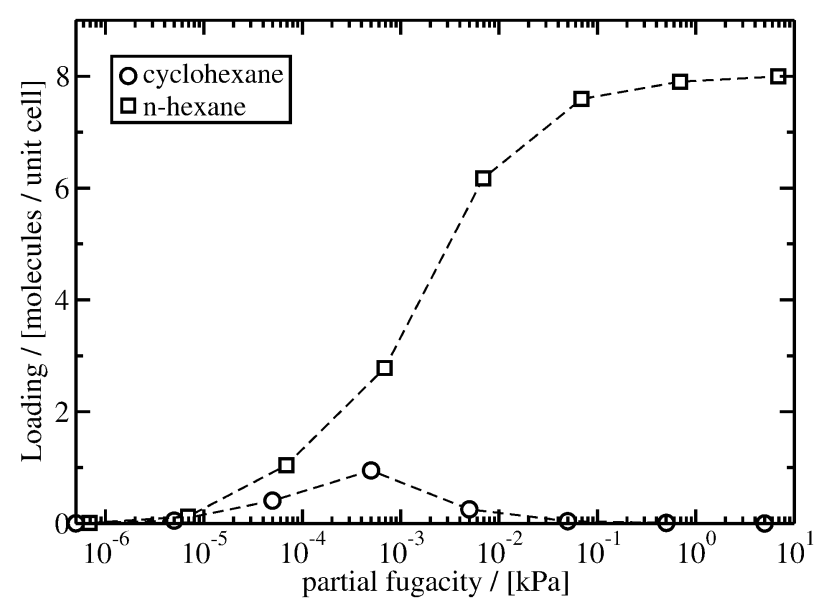

Fig. 11 Simulated adsorption isotherm of an equimolar mixture of cyclohexane and $n$-hexane in MFI-type silica at $T=300 \mathrm{~K}$. The lines serve as a guide to the eye. 


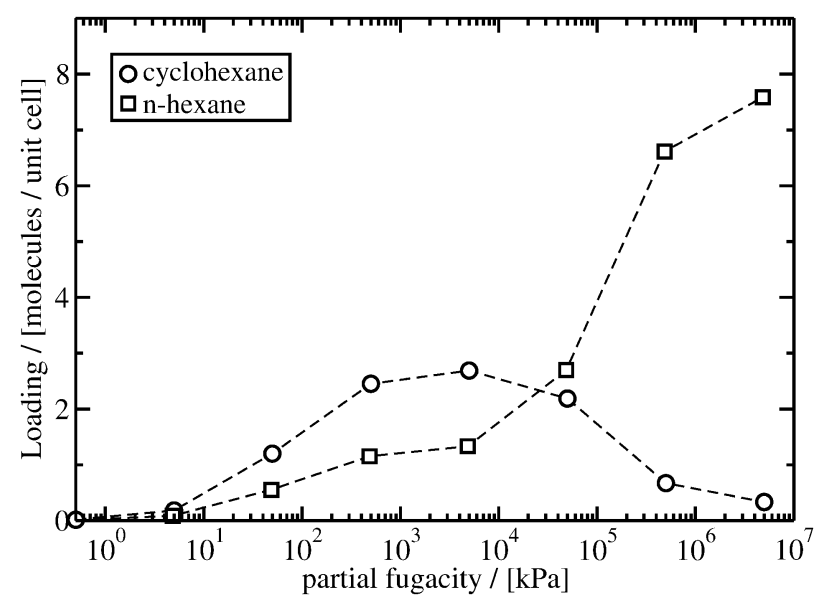

Fig. 12 Simulated adsorption isotherm of an equimolar mixture of cyclohexane and $n$-hexane in MFI-type silica at $T=600 \mathrm{~K}$. The lines serve as a guide to the eye.

have an offset (given in the legend) so that they coincide at 0 at $T=300 \mathrm{~K}$. This figure clearly shows that there is both an energetic and an entropic contribution to the $\Delta \Delta G$, although the latter is the dominant one.

A cause for this difference in temperature dependence of the adsorption entropy can be found when we compare our results to the experimental results for cyclohexane of Harris et al. $(\mathrm{NMR})^{46}$ and Leech et al. (Raman) ${ }^{47}$ and those for $n$-hexane of Jobic et al. (NMR) ${ }^{48}$ and Wang et al. (Raman). ${ }^{49}$ The experiments on cyclohexane reveal a rapid internal reorientation of the molecules at the intersections. As the temperature is increased, the internal reorientation is enhanced, and no extra conformational freedom is lost compared to the gas-phase. This is in contrast to $n$-hexane, where the experiments show that the $n$-hexane molecules have a higher portion of their bonds in the trans conformation compared to the gasphase. ${ }^{48,49}$ This difference is only increased with increasing temperature because the $n$-hexane molecules in the gas-phase will adopt more cis conformations, thus creating a bigger loss in entropy upon adsorption.

In the simulations discussed so far it is assumed, like in the majority of simulation studies on the adsorption in zeolites, that the zeolite framework can be considered rigid. ${ }^{50}$ For molecular dynamics simulations, it is generally believed that the influence of the flexibility is rather small for molecules that are small compared to the pore of the zeolite, ${ }^{51,52}$ but much larger for hydrocarbons that fit tightly into the channels of the zeolite $^{53,54,56}$ or in cation-containing zeolites where vibrations

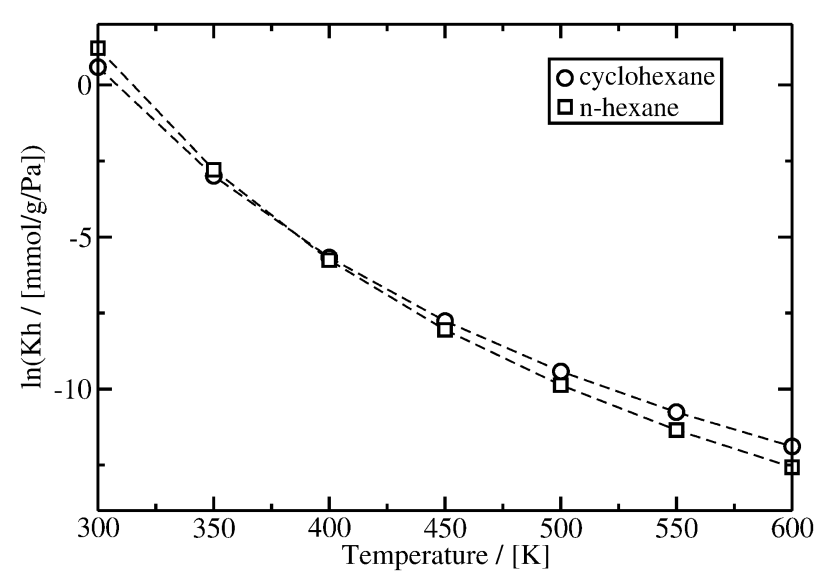

Fig. 13 The natural logarithm of the Henry coefficient as a function of temperature for cyclohexane and $n$-hexane in MFI-type silica. The lines serve as a guide to the eye.

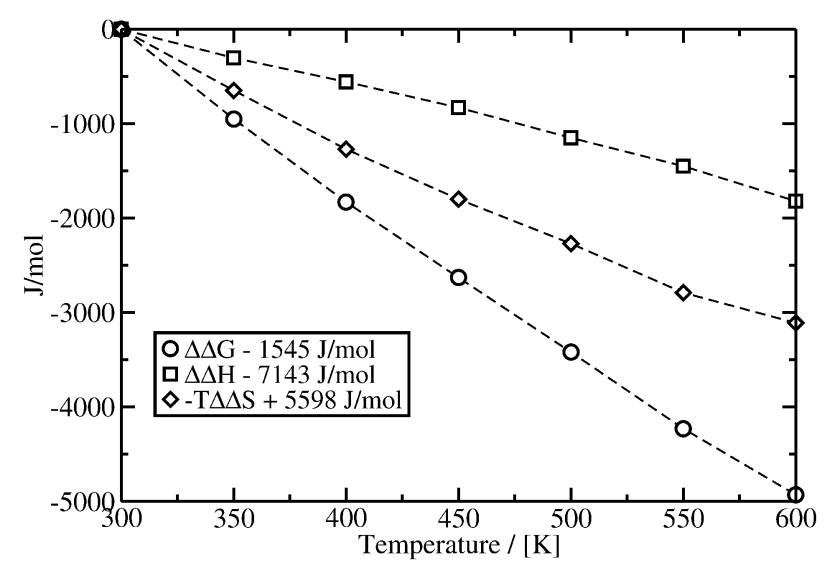

Fig. 14 The difference between the adsorption free energy $(\Delta \Delta G)$, adsorption enthalpy $(\Delta \Delta H)$, and adsorption entropy $(\Delta \Delta S)$ of cyclohexane and $n$-hexane in MFI-type silica at very low loading. To ease viewing, the individual graphs have an offset, given in the legend, so that they coincide at 0 at $T=300 \mathrm{~K}$. The lines serve as a guide to the eye.

of the framework, cation, and adsorbate are strongly coupled. $^{55}$

To investigate the influence of the flexibility of the zeolite framework on the adsorption properties of these rather bulky molecules we performed additional simulations in which the zeolite atoms where allowed to move. The results are presented in Fig. 15. As can been seen, flexibility can have quite some influence on the shape of the adsorption isotherm. When the framework is quite rigid, at $k / k_{\mathrm{B}}=200000 \mathrm{~K}^{-2}$, the adsorption isotherm is very similar to the isotherm in the completely rigid case. At intermediate flexibility, at $k / k_{\mathrm{B}}=$ $20000 \mathrm{~K}^{-2}$, the isotherm shows an inflection at 4 molecules per unit cell. The extra flexibility allows the molecules to also adsorb in the channels. This degree of flexibility compares more or less to the experimentally observed flexibility. ${ }^{28} \mathrm{At}$ $k / k_{\mathrm{B}}=2000 \mathrm{~K}^{-2}$, when the framework is extremely flexible, the adsorption isotherm shows no inflection anymore as the molecules can readily adsorb in the channels.

These simulations using a flexible framework corroborate previous observations ${ }^{29}$ that the framework flexibility has an influence on the adsorption of "bulky" molecules, albeit small for realistic flexibilities. Note however, that we did not change the interaction parameters for these simulations. Re-fitting using the flexible zeolite lattice would probably render slightly different Lennard-Jones parameters, and therefore slightly different isotherms which ultimately may be very similar to the isotherms now simulated with a rigid zeolite lattice.

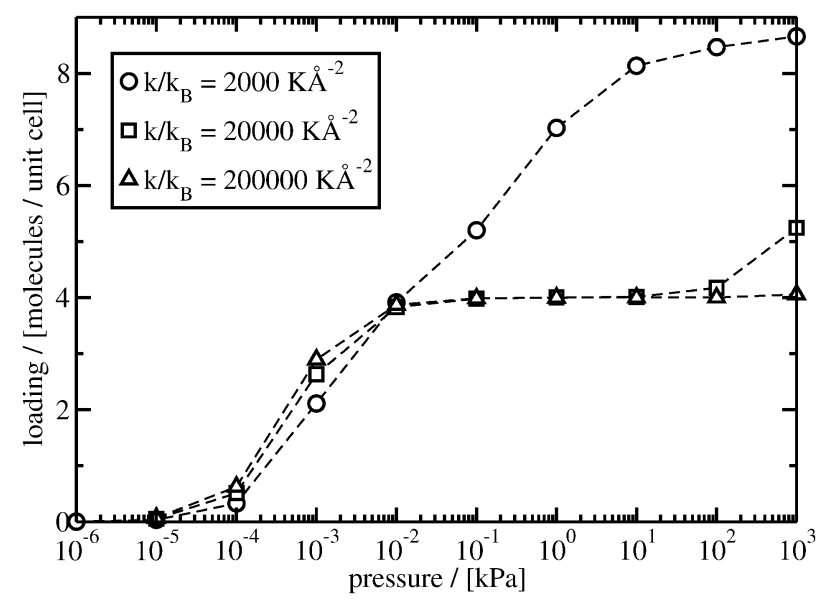

Fig. 15 The influence of zeolite framework flexibility on the adsorption isotherms of cyclohexane in MFI-type silica at $T=300 \mathrm{~K}$. The lines serve as a guide to the eye. 
Added note: After submission of our manuscript a paper was published ${ }^{57}$ with temperature-programmed desorption experiments that show an inflection point for cyclopentane but not for cyclohexane. The conclusions drawn from these experimental results are in good agreement with the principal findings on the inflection behavior of cycloalkanes in MFI-type silica as obtained with our new forcefield.

\section{Conclusions}

In this work we present calculated adsorption isotherms of cycloalkanes and a mixture of cyclohexane and $n$-hexane in MFI-type silica. We have fitted new forcefield parameters for the interactions between the $\mathrm{CH}_{2}$ groups of the cycloalkanes and the silica. These new parameters enable the accurate reproduction of experimentally obtained Henry coefficients, heats of adsorption and adsorption isotherms of cyclohexane in MFI-type silica. Additionally, we present the simulated adsorption isotherms of cyclopentane and cycloheptane in MFI-type silica, for which there is, to the best of our knowledge, no experimental data available. The selectivity in the mixture isotherms shows a temperature dependence at low pressures, while at high pressures entropy effects always favor $n$-hexane adsorption. Furthermore we investigated the influence of zeolite framework flexibility on the adsorption of cyclohexane. We find that, although present, this influence is quite small and comparable to $n$-alkanes.

\section{Acknowledgements}

These investigations are supported in part by the Netherlands Research Council for Chemical Sciences (CW) with financial aid from the Netherlands Technology Foundation, by the Netherlands Organization for Scientific Research (NWO) through PIONIER, by ChevronTexaco, and by the Stichting Nationale Computer Faciliteiten (National Computing Facilities Foundation) through the use of the supercomputer facilities.

\section{References}

1 W. O. Haag, in Zeolites and Related Microporous Materials State of the Art 1994, Studies in Surface Science and Catalysis, eds. J. Weitkamp, H. G. Karge, H. Pfeifer and W. Hölderich, Elsevier, Amsterdam, 1994, vol. 84, p. 1375.

2 T. L. M. Maesen, M. Schenk, T. J. H. Vlugt, J. P. de Jonge and B. Smit, J. Catal., 1999, 188, 403.

3 M. Schenk, B. Smit, T. J. H. Vlugt and T. L. M. Maesen, Angew. Chem. Int. Ed., 2001, 40, 736.

4 M. Schenk, S. Calero, T. L. M. Maesen, L. L. van Benthem, M. G. Verbeek and B. Smit, Angew. Chem. Int. Ed., 2002, 41, 2500.

5 H. Stach, H. Thamm, J. Jänchen, K. Fiedler and W. Schirmer, in New Developments in Zeolite Science and Technology, Proceedings of the 6th International Zeolite Conference, eds. Olsen and D. Bisio, A. Butterworth, Guildford, UK, 1984, p. 225.

6 R. L. June, A. T. Bell and D. N. Theodorou, J. Phys. Chem., 1992 96, 1051 .

7 B. Smit and T. L. M. Maesen, Nature, 1995, 374, 42.

8 P. I. Pohl, G. S. Heffelfinger and D. M. Smith, Mol. Phys., 1996, 89, 1725.

9 R. C. Runnebaum and E. J. Maginn, J. Phys. Chem. B, 1997, 101 6394.

10 R. Krishna, B. Smit and T. J. H. Vlugt, J. Phys. Chem. A, 1998, $102,7727$.

11 T. J. H. Vlugt, W. Zhu, F. Kapteijn, J. A. Moulijn, B. Smit and R. Krishna, J. Am. Chem. Soc., 1998, 120, 5599.

12 T. J. H. Vlugt, R. Krishna and B. Smit, J. Phys. Chem. B, 1999 103, 1102.

13 N. G. Leonidas and D. N. Theodorou, J. Phys. Chem. B, 1999 103, 3380.

14 E. B. Webb III, G. S. Grest and M. Mondello, J. Phys. Chem. B, 1999, 103, 4949 .
15 M. D. Macedonia and E. J. Maginn, Fluid Phase Equilib., 1999, 160, 19.

16 M. D. Macedonia, D. D. Moore, E. J. Maginn and M. M. Olken, Langmuir, 2000, 16, 3823.

17 D. Schuring, A. P. J. Jansen and R. A. van Santen, J. Phys. Chem. $B, 2000,104,941$.

18 A. H. Fuchs and A. K. Cheetham, J. Phys. Chem. B, 2001, 105, 7375 .

19 K. Makrodimitris, G. K. Papadopoulos and D. N. Theodorou, J. Phys. Chem. B, 2001, 105, 777.

20 H. L. Tepper and W. J. Briels, J. Chem. Phys., 2002, 116, 9464.

21 S. Furukawa, C. McCabe, T. Nitta and P. T. Cummings, Fluid Phase Equilib., 2002, 194, 309.

22 M. Chandross, E. B. Webb III, G. S. Grest, M. G. Martin, A. P. Thompson and M. W. Roth, J. Phys. Chem. B, 2001, 105, 5700.

23 P. Pascual and Boutin, Phys. Chem. Chem. Phys., 2004, 6, 2015.

24 A. Gupta, L. A. Clark and R. Q. Snurr, Langmuir, 2000, 16, 3910.

25 M. Murthi and R. Q. Snurr, Langmuir, 2004, 20, 2489.

26 J. P. Fox, V. Rooy and S. P. Bates, Microporous Mesoporous Mater., 2004, 69, 9.

27 J. P. Fox and S. P. Bates, J. Phys. Chem., 2004, 108, 17136.

28 P. Demontis, G. B. Suffritti, S. Quartieri, E. S. Fois and A. Gamba, J. Phys. Chem., 1988, 92, 867.

29 T. J. H. Vlugt and M. Schenk, J. Phys. Chem. B, 2002, 106, 12757.

30 J.-M. B. Ndjaka, G. Zwanenburg, B. Smit and M. Schenk, Microporous Mesoporous Mater., 2004, 68, 37.

31 B. Neubauer, A. Boutin, B. Tavitian and A. H. Fuchs, Mol. Phys., 1999, 97, 769.

32 D. Frenkel and B. Smit, Understanding Molecular Simulation: from Algorithms to Applications, Academic Press, San Diego, 2nd edn., 2002.

33 J. R. Errington and A. Z. Panagiotopoulos, J. Chem. Phys., 1999, 111, 9731.

34 C. D. Wick, M. G. Martin and J. I. Siepmann, J. Phys. Chem., 2000, 104, 8008 .

35 S. Chempath, L. A. Clark and R. Q. Snurr, J. Chem. Phys., 2003, 118, 7635.

36 K. Esselink, L. D. J. C. Loyens and B. Smit, Phys. Rev. E, 1995, 51, 1560.

37 W. Zhu, J. M. van de Graaf, L. J. P. van de Broeke, F. Kapteijn and J. A. Moulijn, Ind. Eng. Chem. Res., 1998, 37, 1934.

38 M. S. Sun, D. B. Shah, H. H. Xu and O. Talu, J. Phys. Chem., 1998, 102, 1466.

39 D. Dubbeldam, S. Calero, T. J. H. Vlugt, R. Krishna, T. L. M. Maesen and B. Smit, J. Phys. Chem. B, 2004, 108, 12301.

40 M. G. Martin and J. I. Siepmann, J. Phys. Chem. B, 1998, 102, 2569.

41 C. L. Cavalcante and D. M. Ruthven, Ind. Eng. Chem. Res., 1995, 34, 177.

42 L. Song and L. V. C. Rees, Microporous Mesoporous Mater., 2000, 35-36, 301.

43 B. Smit, J. Phys. Chem. B, 1995, 99, 5597.

44 D. Dubbeldam, S. Calero, T. J. H. Vlugt, R. Krishna, T. L. M. Maesen, E. Beerdsen and B. Smit, Phys. Rev. Lett., 2004, 93, 88302 .

45 R. Krishna, B. Smit and S. Calero, Chem. Soc. Rev., 2002, 31, 185.

46 A. E. Aliev and K. D. M. Harris, J. Phys. Chem. A, 1997, 101, 4541 .

47 Y. Huang and J. Leech, J. Phys. Chem. A, 2001, 105, 6965.

48 A. G. Stepanov, A. A. Shubin, M. V. Luzgin, T. O. Shegai and H. Jobic, J. Phys. B, 2003, 107, 7095.

49 Y. Huang and H. Wang, Langmuir, 2003, 19, 9706.

50 A. G. Bezus, A. V. Kiselev, A. A. Lopatkin and P. Q. Du, J. Chem. Soc., Faraday Trans. 2, 1978, 74, 367.

51 S. Fritzche, M. Wolfsberg, R. Haberlandt, P. Demontis, G. B. Suffritti and A. Tilocca, Chem. Phys. Lett., 1999, 296, 253.

52 D. I. Kopelevich and H. C. Chang, J. Chem. Phys., 2001, 114, 3776.

53 T. R. Forester and W. Smith, J. Chem. Soc., Faraday Trans., 1997, 93, 3249.

54 A. Bouyermaouen and A. Bellemans, J. Chem. Phys., 1998, 108, 2170 .

55 F. Jousse, S. M. Auerbach and D. P. Vercauteren, J. Phys. Chem. $B, 2000,104,2360$.

56 F. Leroy, B. Rousseau and A. H. Fuchs, Phys. Chem. Chem. Phys., 2004, 6, 775.

57 M. Dai, H. Ban, X. Zhang, L. Song and Z. Sun, J. Fuel Chem. Technol. (Taiyuan, People's Repub. China), 2005, 33, 117. 